\title{
Is infrasound perceived by the auditory system through distortions?
}

\author{
Holger Joost ${ }^{1, *}$, Björn Friedrich ${ }^{2}$, Jesko L. Verhey $^{2}$, and Thomas Fedtke ${ }^{1}$ \\ ${ }^{1}$ Physikalisch-Technische Bundesanstalt, Bundesallee 100, 38116 Braunschweig, Germany \\ ${ }^{2}$ Department of Experimental Audiology, Otto von Guericke University Magdeburg, Leipziger Str. 44, 39120 Magdeburg, Germany
}

Received 18 May 2020, Accepted 1 December 2020

\begin{abstract}
Several studies showed that the human auditory system is sensitive to infrasound. The present study investigated if non-linear processes within the ear generate distortion products, which may explain the auditory sensitivity to infrasound. Pure-tone infrasound stimuli were presented to the ears of 16 listeners with a low-distortion sound reproduction system via an ear insert. Simultaneously, the sound in the ear canal was recorded with a high-sensitivity probe microphone. In addition, detection thresholds in quiet for these stimuli were determined in all listeners. All of the listeners showed distortion products, which were detected by analyzing the sound recorded in their ear canals during infrasound stimulation, for at least one of the signal frequencies. The sound pressure levels of the distortion products were well below the reference thresholds at the corresponding frequencies. For each signal frequency, the listeners' detection thresholds in cases with eargenerated distortion products did not differ significantly from those in cases without. Thus, the present data do not support the hypothesis that distortion products play a major role in the auditory perception of infrasound.
\end{abstract}

Keywords: Infrasound perception, Auditory distortion products, Low-distortion reproduction system

\section{Introduction}

In the light of a steadily increasing number of reports on infrasound exposure, it is important to understand how humans perceive infrasound, which is defined as sound with frequencies below $20 \mathrm{~Hz}$ [1]. Several studies indicate that the human auditory system is sensitive to infrasound down to at least $2 \mathrm{~Hz}$ (e.g., [2-4]; for a review, see [5]). They show that an auditory detection threshold can be determined also for infrasound stimuli, provided that they have sufficiently high Sound Pressure Levels (SPLs). Recent studies on human brain activation [6-8] show that the brain responds to infrasound that is being presented to the ear, at threshold and at higher loudness levels. Two of them [7, 8] show an activation of the primary auditory cortex. This further supports the view that infrasound is processed by the auditory system. Until now, however, the mechanisms underlying this auditory infrasound perception have not yet been fully understood.

A hypothesis is that non-linear processes within the ear generate distortion products, in the following referred to as ear-generated distortion products, that contribute to the perception of infrasound [3]. The high SPLs required to perceive infrasound may generate such distortion products by means of a number of mechanisms: For instance, elastic structures of the ear may be driven outside their linear

*Corresponding author: holger. joost@ptb.de range to such an extent that higher harmonics are generated. The non-harmonic displacement of the eardrum would produce measurable distortion products in the ear canal. Furthermore, non-linear behavior of middle-ear structures could also generate distortion products in the ear canal. In general, distortion products in the audio-frequency range that have SPLs above the listeners' individual thresholds could contribute to infrasound perception. A different mechanism is that distortion products are generated within the cochlea, e.g., by active outer hair cell motility, and transmitted back to the ear canal where they can be measured like Otoacoustic Emissions (OAEs, [9]). Distortion products generated in this way could contribute to infrasound perception even if the SPLs in the ear canal were well below the listeners' individual thresholds, since these distortion products are attenuated on their way from the cochlea to the ear canal. The existence of audio-frequency ear-generated distortion products may also explain the recent finding that simultaneously presented audio sound increases thresholds of infrasound [10]: Audio sound might, at least partially, mask ear-generated distortion products.

The aim of the present study was to test the hypothesis of audible ear-generated distortion products by examining the sound field in the human ear canal for higher harmonic distortions during the stimulation with supra-threshold pure-tone infrasound. To this end, a custom-made Low-Distortion Sound Reproduction System (LDREPS) was used for presenting the stimuli and recording the sound in the 
ear canal [11]. Since this study focused on the perception of infrasound by the auditory system, stimuli were delivered exclusively to the ear canal by means of the LDREPS ear insert. To avoid false positive results when analyzing the presence of ear-generated distortion products, it is mandatory that both, the infrasound presentation system and the ear-canal sound recording system, do not generate pronounced harmonic distortions, in the following referred to as technical distortions. These were determined in an occluded-ear simulator prior to the measurements in human ear canals. In each of the listeners, also the SPLs at the behavioral detection thresholds in quiet for the infrasound stimuli were determined. For simplicity, these will be referred to as thresholds in the following. They were compared to literature data ([5] for infrasound frequencies; [12] for audio frequencies), which will be referred to as reference thresholds in the following. To test a possible contribution of distortion products to infrasound detection, the thresholds were related to the analysis of the sound field in the ear canal and to the physiological background noise therein.

\section{Methods}

\subsection{The Low-Distortion Sound Reproduction System (LDREPS)}

Figure 1 shows key parts of the sound reproduction system, LDREPS. In the top of the figure, there are two commercially available RadioEar DD45 audiometric earphone transducers (RadioEar, Denmark), which are mounted in air-sealed aluminum housings, each with a sound outlet in the front plate. Sound tubes with a length of $25 \mathrm{~cm}$ connect the sound outlets to the ear insert of an Etymotic ER-10B+ low-noise microphone system (Etymotic Research Inc., USA, bottom of the figure). The volume that is enclosed by the membrane of the earphone transducers, the tubes, and the ear is so small that it acts as a pressure chamber for low frequencies, enabling acoustic stimulation with the DD45 earphone transducers down to infrasound frequencies. The LDREPS was designed with two DD45 transducers to allow the user to transmit audio-sound and infrasound stimuli via separate channels. Since the present study was only using pure-tone infrasound signals, only one of the transducers was driven. Still, the second DD45 cartridge was acoustically connected, since it constitutes an integral part of the acoustically characterized LDREPS. The DD45 transducer was driven by a custom-made dual-channel headphone amplifier, based on an LPA-2S board (Funk Tonstudiotechnik, Germany). The board was modified by the manufacturer for the special purpose of driving the low-impedance $(10 \Omega)$ headphone transducer down to $2 \mathrm{~Hz}$ with a maximum power of $500 \mathrm{~mW}$. This headphone amplifier offers high output power combined with low noise and minimum harmonic distortion (THD + N: $1 \mathrm{kHz}$ signal $0.0013 \%$; $10 \mathrm{~Hz}$ signal $0.0006 \%$; both at $500 \mathrm{~mW} / 10 \Omega$ ).

The LDREPS was specially developed for presenting signals with very low distortion in the frequency range

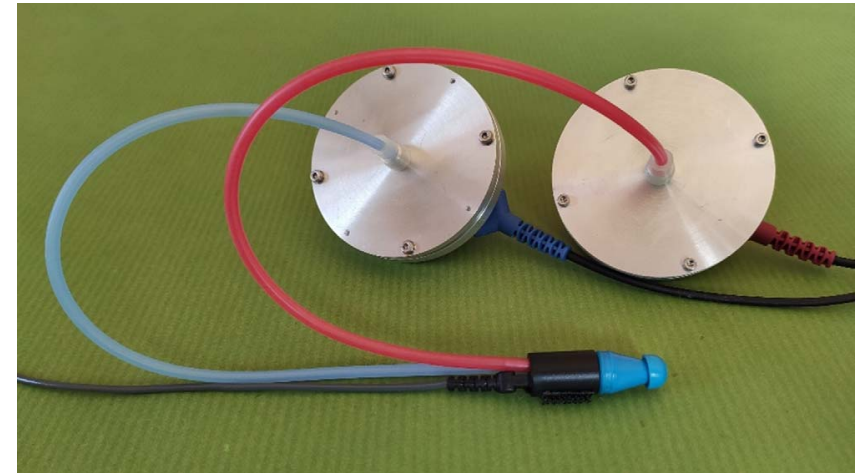

Figure 1. Picture of the two DD45 earphone transducers (top) coupled with the Etymotic ER-10B + system (bottom) via two tubes, as part of the LDREPS.

from $4 \mathrm{~Hz}$ to $4 \mathrm{kHz}$ and for SPLs that are equivalent to a loudness level of 40 phon. The system used in the present study was a slightly modified version of the one described in [11]: A low-pass filter (second order, $-3 \mathrm{~dB}$ cutoff frequency: $30 \mathrm{~Hz}$ ) was inserted in the signal path between amplifier and transducer for safety reasons, ensuring that high SPLs can be presented in the infrasound range while the levels in the audio-frequency range are limited. This modified version was already used to measure the effect of number of spectral components on the infrasound detection threshold [13].

\subsection{Analysis of technical distortions}

To ensure that potential distortion products in the ear canal are not generated by the LDREPS itself, it is crucial to first quantify the technical distortions of the system. These were determined using the setup shown in Figure 2. The infrasound emitted from the DD45 earphone transducer was guided through a sound tube to the Etymotic ER-10B + ear insert. The ear insert was connected to a B\&K 4157 occluded-ear simulator (Brüel \& Kjær, Denmark) by means of the adapter DB-2012, which simulates the external ear canal. The Etymotic ER-10B + microphone measured the sound in the occluded-ear simulator. The microphone signal was amplified by the dedicated microphone preamplifier and was then forwarded to one channel of the APx555 audio analyzer (Audio Precision, USA). The APx555 audio analyzer also provided the pure-tone test signals, which were fed into the earphone amplifier Funk LPA$2 \mathrm{~S}$ driving the DD45 transducer through a custom-built low-pass filter.

The DD45 earphone transducers, the Etymotic ER-10B + ear insert, and the B\&K 4157 occluded-ear simulator were placed in a double-walled sound-insulated booth. The ER-10B + microphone was calibrated using the ear simulator as a reference (see [11]). It was not overloaded by the high infrasound pressure levels and, at the same time, showed enough sensitivity and sufficiently low noise to measure distortion products for both, infrasound and audio sound, while high-level infrasound was present (see Fig. 1 in [11]). Magnitude spectra were measured in silence 


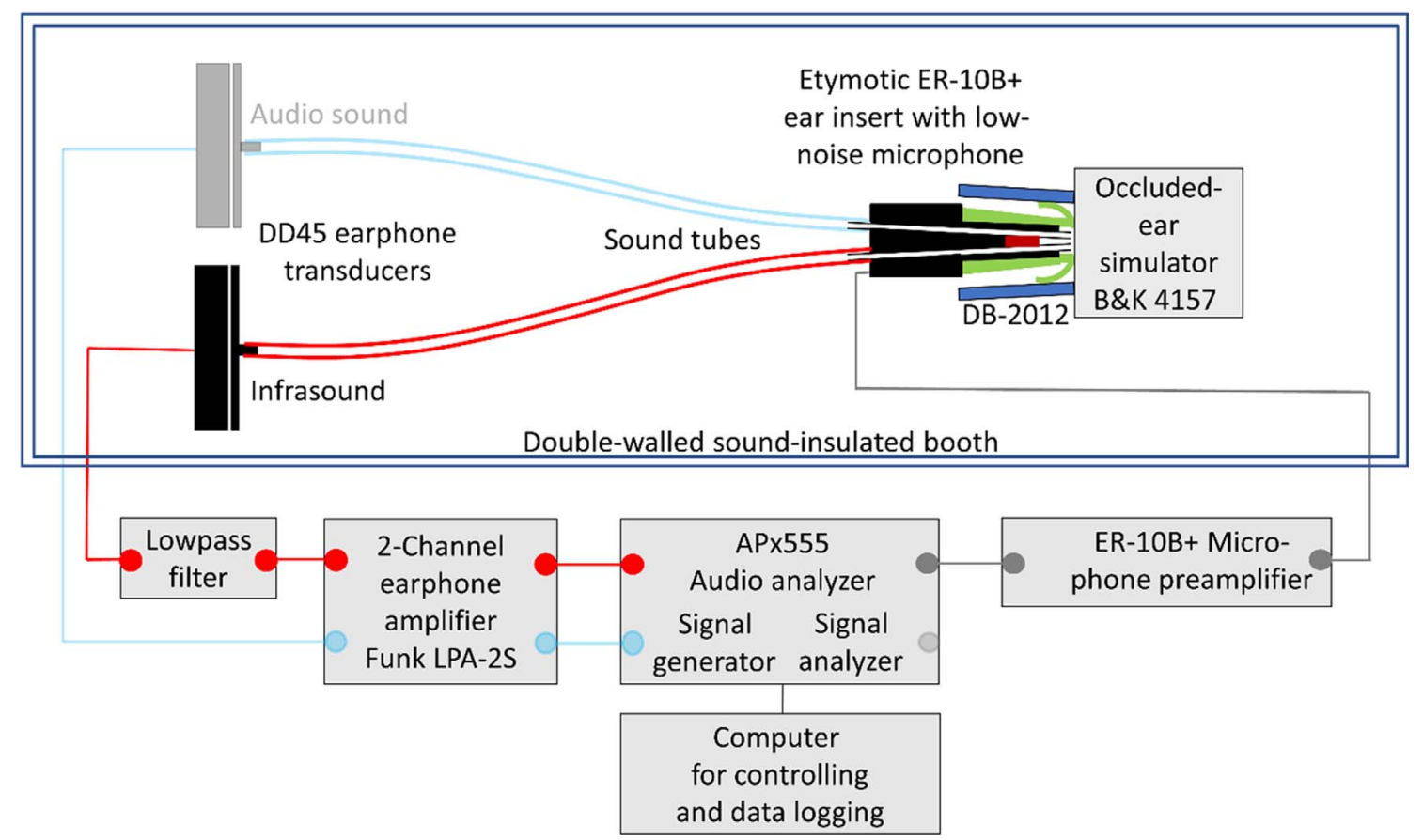

Figure 2. Block diagram of the setup for measuring technical distortions of the LDREPS. Red and gray connecting lines highlight the parts that were used in the present study. The second channel (light blue connecting lines) enables the LDREPS to present an additional audio-sound stimulus (not used in this study).

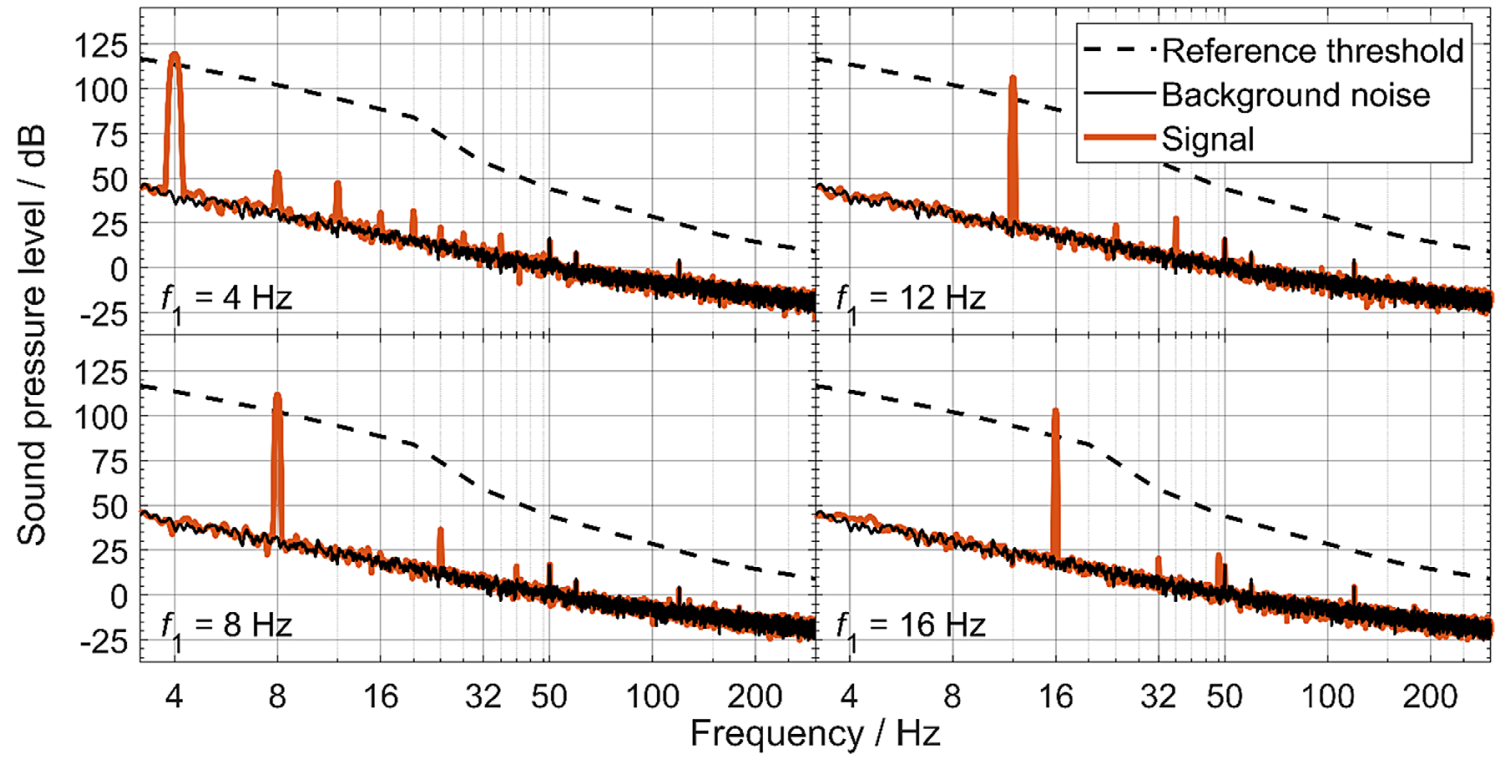

Figure 3. Magnitude spectra (orange solid lines) produced by the LDREPS in the occluded-ear simulator and measured with the ER10B + microphone. Each panel shows the averaged spectrum for a pure-tone signal at a level above its reference threshold. The signal frequency, $f_{1}$, is indicated in the bottom left corner of the panel. For comparison, the spectrum of a background noise measurement is also shown (dark gray solid line). In addition, the reference thresholds are shown as a black dashed line. The frequency spectra are limited to the frequency range from $3 \mathrm{~Hz}$ to $300 \mathrm{~Hz}$.

and for pure tones with frequencies of $4,8,12$, and $16 \mathrm{~Hz}$. The recorded signal was analyzed by the APx555 audio analyzer, which calculated magnitude spectra in blocks of about $20 \mathrm{~s}$ with a resolution of $46 \mathrm{mHz}$ using a flat-top window. The spectra were then averaged. Each panel of Figure 3 shows an averaged spectrum (average of five; orange solid lines) of the signal that was recorded in the B\&K 4157 occluded-ear simulator by means of the ER-10B + microphone. In each panel, the frequency spectrum is limited to the frequency range from $3 \mathrm{~Hz}$ to $300 \mathrm{~Hz}$. The SPLs of the pure-tone signals were $120 \mathrm{~dB}$ for the $4 \mathrm{~Hz}$ tone, $111 \mathrm{~dB}$ for $8 \mathrm{~Hz}, 106 \mathrm{~dB}$ for $12 \mathrm{~Hz}$, and 
Table 1. Parameters of stimuli used for threshold measurements.

\begin{tabular}{lcccc}
\hline Stimulus frequency $[\mathrm{Hz}]$ & Interval duration $[\mathrm{ms}]$ & Ramp duration $[\mathrm{ms}]$ & Starting SPL $[\mathrm{dB}]$ & Maximum SPL $[\mathrm{dB}]$ \\
\hline 4 & 2500 & 750 & 117 & 121.3 \\
8 & 1250 & 375 & 106 & 112.5 \\
12 & 1125 & 250 & 100 & 106.9 \\
16 & 1000 & 187.5 & 96 & 103.8 \\
\hline
\end{tabular}

$103 \mathrm{~dB}$ for $16 \mathrm{~Hz}$. They corresponded to loudness levels that were slightly below 40 phon (see [5]). Even at the SPLs used in the present study, the levels of the technical distortions were at least $20 \mathrm{~dB}$ below the reference thresholds (dashed lines in Fig. 3). Note that these distortions were more prominent than those shown in [11]. This is due to the additional non-linearity of the low-pass filter that was inserted in the signal path.

\subsection{Measurement of ear-generated distortion products}

The stimuli and the setup were essentially the same as the one used to measure technical distortions of the LDREPS (see Fig. 2) with the only difference that the $\mathrm{ER} 10 \mathrm{~B}+$ ear insert was positioned in the left ear canal of the listener, instead of in the occluded-ear simulator. The listeners were seated in a double-walled sound-insulated booth. The signal in the ear canal was recorded by means of the ER-10B + microphone in silence and for pure tones with frequencies of $4,8,12$, and $16 \mathrm{~Hz}$. To reduce interference by body-borne noise in the recorded signal, listeners were asked to suspend their deglutition and masticatory movements as much as possible, and to avoid any unnecessary motion during the two-minute measurement phases. As for the analysis of the technical distortions, the recorded signal was analyzed by the APx555 audio analyzer, which calculated averaged magnitude spectra with a resolution of $46 \mathrm{mHz}$. If a magnitude spectrum was interfered by unwanted noises, it was discarded, and the measurement was repeated to get the specified number of averages for the spectrum. During each measurement, the proper fit of the ear insert was controlled in-situ by the level of the fundamental.

\subsection{Measurement of thresholds}

Thresholds were measured for pure tones with frequencies of 4, 8, 12, and $16 \mathrm{~Hz}$ in the same ear in which the sound was recorded with the high-sensitivity ER-10B + microphone during supra-threshold infrasound presentation. As in [4] and [13], the duration of the ramps and total duration of the signals (including raised cosine on- and offset ramps) depended on frequency (see second and third column of Tab. 1).

A three-interval, three-alternative forced-choice procedure with a one-up two-down rule was used, which leads to a detectability at threshold of $70.7 \%$ [14]. The intervals of a trial were separated by $250 \mathrm{~ms}$ of silence. During the presentation of a trial, the timings of the intervals were highlighted on a screen, which was positioned in front of the listener. One randomly chosen interval contained the signal.

The listener was requested to press a key on a hand-held keyboard to indicate which of the intervals contained the signal. The adaptive procedure started at an SPL that corresponded to a loudness level of $\sim 20$ phon (see [5]). The starting SPLs (in $\mathrm{dB}$ ) are shown in the fourth column of Table 1. The initial step size was $4 \mathrm{~dB}$ and was halved after each upper reversal until the final step size of $1 \mathrm{~dB}$ was reached. The run continued with the final step size for four reversals. The mean value of the SPLs at these four reversals was taken as a threshold estimate. For each of the four pure-tone stimuli, thresholds were measured twice. The mean value of these two threshold estimates was taken as the final threshold estimate.

The adaptive procedure was controlled by a MATLAB program. All signals were generated with a sampling rate of $96 \mathrm{kHz}$ and a resolution of 24 bits. The digital signals were converted to analog signals via an external sound card (RME Fireface, Haimhausen, Germany) and presented to the ear through the LDREPS. To spot-check the proper fit of the ear insert, the sound pressure levels of the puretone signals, which had been calibrated in a B\&K 4157 occluded-ear simulator (Brüel \& Kjær, Denmark), were measured in-situ by means of the ER-10B + microphone prior to or after the listening tests. Before the actual threshold measurements, listeners had a short practice run with a $16 \mathrm{~Hz}$ stimulus to familiarize with the experiment, in which communication with the experimenter was possible.

The thresholds of the listeners were subdivided into groups based on the presence or absence of distortions. Differences between median thresholds of these groups were quantified using the nonparametric two-sided Wilcoxon rank-sum test. The significance level was set to $\alpha=0.05$.

\subsection{Listeners}

Sixteen listeners (9 female, 7 male) between 19 and 33 years $($ median $=22$ years) participated in the experiments. All were paid volunteers. Prior to the experiment, the functionality of their hearing was tested. Their audiometric hearing levels of the ear that was used for the infrasound experiment were lower than or equal to $15 \mathrm{~dB}$ for all audiometric frequencies between $125 \mathrm{~Hz}$ and $4 \mathrm{kHz}$. All participants were otologically normal as confirmed by a questionnaire for hearing testing (Annex A of ISO 389-9 [15]) and by otoscopic examination. They were instructed on the goal of the study and on the anonymized usage of their data according to the General Data Protection Regulation (GDPR) of the European Union. The Declaration of 


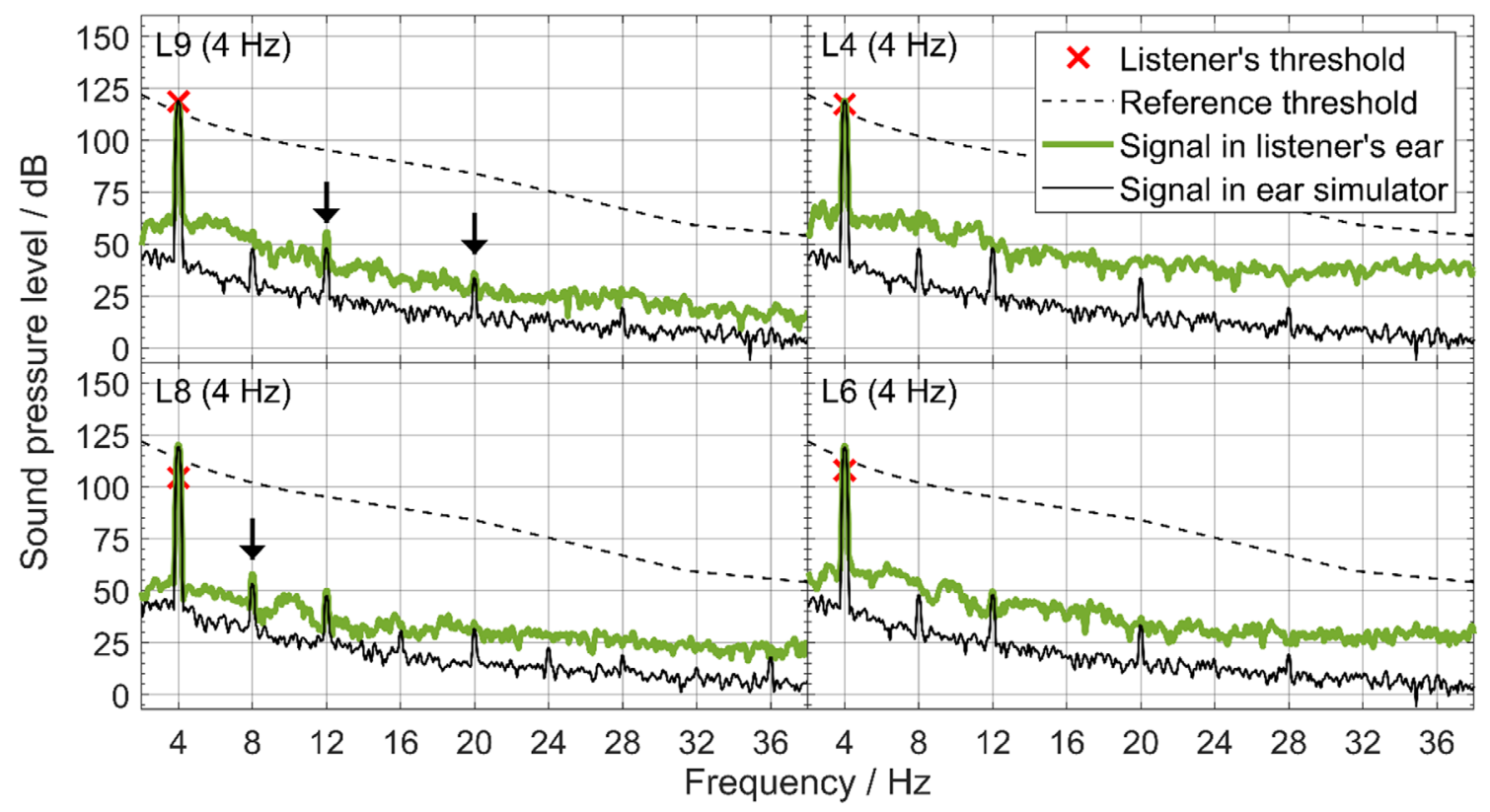

Figure 4. Magnitude spectra for a $4 \mathrm{~Hz}$ infrasound, measured with the low-noise ER-10B + microphone, in the ear canal of four listeners (green solid lines) and the corresponding spectra in the B\&K 4157 occluded-ear simulator (black solid lines). Each panel shows the result of one listener. In all panels, the spectra are limited to the frequency range from $2 \mathrm{~Hz}$ to $38 \mathrm{~Hz}$. The reference thresholds are shown as a black dashed line, the individual thresholds as red crosses. Black arrows indicate ear-generated distortion products, which were identified by means of criteria described in the text (see also Fig. 5).

Helsinki was adhered to in all our measurements. Approval was obtained from the local ethics committee (Medical faculty of the Otto von Guericke University Magdeburg, ethics application 79/17).

\section{Results}

\subsection{Ear-generated distortion products}

Figure 4 shows examples of magnitude spectra recorded in the ears of four listeners (green solid lines). Each panel shows the result for a $4 \mathrm{~Hz}$ signal of one listener and the corresponding spectrum measured in the B\&K 4157 occludedear simulator (black solid lines). Since in the experiment two realizations of the low-pass filter were used, the spectrum was not the same for all listeners. In each panel, the frequency spectra are limited to the frequency range from $2 \mathrm{~Hz}$ to $38 \mathrm{~Hz}$. The background noise levels in the listeners' ears are higher than the background noise levels in the ear simulator.

The average spectra of all listeners were analyzed with respect to peaks at the higher harmonic frequencies $f_{2}$ to $f_{9}$ of the respective signal frequency $f_{1}$. Since the spectra were noisy, a criterion had to be defined to distinguish an ear-generated peak at a higher harmonic frequency from a random fluctuation of the power spectral density. In addition, it was necessary to ensure that the peak did not represent just a technical distortion component, which led to a second criterion. Figure 5 visualizes the two criteria that were used to detect an ear-generated distortion product. It shows a section of the spectrum of the $4 \mathrm{~Hz}$ signal measured in the ear of example listener L8 (whose $4 \mathrm{~Hz}$ spectrum is also shown in the bottom left corner of Fig. 4).

Both criteria are based on ratios of power spectral densities. The first criterion calculates the ratio between the tone and surrounding-noise power spectral densities (cf. [16], Eq. (1)). To assess the power density of the tone, the power is determined in a narrow band around its harmonic frequency. The width of the band is $\Delta f_{t}=0.4 \mathrm{~Hz}$. This is shown in Figure 5 for the higher harmonic frequencies $8 \mathrm{~Hz}, 12 \mathrm{~Hz}$, and $16 \mathrm{~Hz}$ with dark gray areas. Since in real-world settings, this frequency band contains the tone and the background noise (see [16]), the respective power will be referred to as $P_{\mathrm{t}+\mathrm{n}}(\mathrm{t}+\mathrm{n}$ for tone and noise) in the following. The power spectral density is then

$$
\varrho_{\mathrm{t}+\mathrm{n}}=\frac{P_{\mathrm{t}+\mathrm{n}}}{\Delta f_{\mathrm{t}}}
$$

The power spectral density of the surrounding noise is determined in a wider frequency band with a bandwidth $\Delta f_{\text {tot }}=2.4 \mathrm{~Hz}$. To get an estimate of the power of this surrounding noise (i.e., in the light gray areas of Fig. 5), the difference between the total power $P_{\text {tot }}$ in this wider band and $P_{\mathrm{t}+\mathrm{n}}$ was calculated. The power spectral density $\varrho_{\mathrm{n}}$ of the surrounding noise is then

$$
\varrho_{\mathrm{n}}=\frac{P_{\mathrm{tot}}-P_{\mathrm{t}+\mathrm{n}}}{\Delta f_{\mathrm{tot}}-\Delta f_{\mathrm{t}}} .
$$

To be classified as a peak, the ratio of these two power spectral densities should be at least two. When expressed as a level difference $\Delta L_{\mathrm{tn}}$, this is equivalent to 


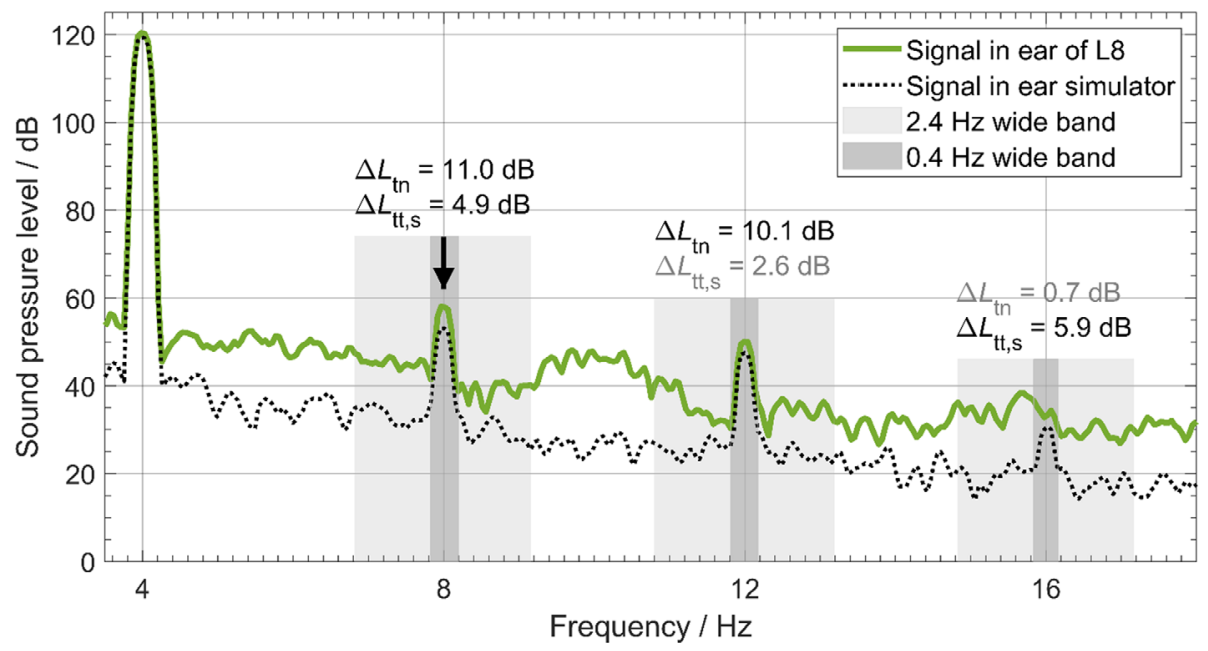

Figure 5. Example magnitude spectra to visualize the criteria for the detection of ear-generated distortions. To this end, a portion of the spectra of the bottom left panel of Figure 4 is redrawn. The dark gray boxes indicate the $0.4 \mathrm{~Hz}$ wide frequency bands around the higher harmonics that were used to derive their power spectral densities. The light gray boxes indicate the $2.4 \mathrm{~Hz}$ wide frequency bands around the higher harmonic that were used to estimate the power spectral density of the noise. From these two quantities, a tone-to-noise ratio was calculated and expressed as a level difference $\Delta L_{\mathrm{tn}}$. In addition, the ratio between the power in the narrow frequency band around the higher harmonics between the spectrum recorded in the listener's ear and that recorded in the occluded-ear simulator was computed and expressed as a level difference $\Delta \mathrm{L}_{\mathrm{tt}, \mathrm{s}}$. Based on these measures, it was decided whether an ear-generated distortion product is present (indicated by the black arrow) or not (see text for details).

$\Delta L_{\mathrm{tn}}=10 \lg \left(\frac{P_{\mathrm{t}+\mathrm{n}}}{\Delta f_{\mathrm{t}}}\right) \mathrm{dB}-10 \lg \left(\frac{P_{\mathrm{tot}}-P_{\mathrm{t}+\mathrm{n}}}{\Delta f_{\mathrm{tot}}-\Delta f_{\mathrm{t}}}\right) \mathrm{dB}>3 \mathrm{~dB}$

This criterion was applied to the higher harmonics $f_{2}$ to $f_{9}$ of the signal frequency $f_{1}$.

The second criterion compares the spectrum in the ear canal with the spectrum in the occluded-ear simulator. For both spectra, the power spectral densities in the narrow frequency band $\left(\Delta f_{\mathrm{t}}=0.4 \mathrm{~Hz}\right)$ around the higher harmonic frequency were determined. The ratio of these two quantities should be at least two. As a level difference $\Delta L_{\mathrm{tt}, \mathrm{s}}$, this is equivalent to

$$
\Delta L_{\mathrm{tt}, \mathrm{s}}=10 \lg \left(\frac{P_{\mathrm{t}+\mathrm{n}}}{P_{\mathrm{t}+\mathrm{n}, \mathrm{s}}}\right) \mathrm{dB}>3 \mathrm{~dB},
$$

where $P_{\mathrm{t}+\mathrm{n}, \mathrm{s}}$ is the power in the narrow band around the higher harmonic frequency for the occluded-ear simulator, indicated by the additional subscript "s". Only if both criteria were fulfilled, the distortion component was classified as being an ear-generated distortion product.

Regarding the example shown in Figure 5 , for the $8 \mathrm{~Hz}$ component, $\Delta L_{\mathrm{tn}}$ is $11.0 \mathrm{~dB}(>3 \mathrm{~dB})$ and $\Delta L_{\mathrm{tt}, \mathrm{s}}$ is $4.9 \mathrm{~dB}$ $(>3 \mathrm{~dB})$. Hence, this component is, according to the criteria of the present study, an ear-generated distortion product. The $12 \mathrm{~Hz}$ component fulfills the first criterion $\left(\Delta L_{\mathrm{tn}}=10.1 \mathrm{~dB}>3 \mathrm{~dB}\right)$, but not the second one $\left(\Delta \mathrm{L}_{\mathrm{tt}, \mathrm{s}}=\right.$ $2.6 \mathrm{~dB}<3 \mathrm{~dB}$ ). The $16 \mathrm{~Hz}$ component does not fulfill the first criterion $\left(\Delta L_{\mathrm{tn}}=0.7 \mathrm{~dB}<3 \mathrm{~dB}\right)$, but fulfills the second one $\left(\Delta L_{\mathrm{tt}, \mathrm{s}}=5.9 \mathrm{~dB}>3 \mathrm{~dB}\right)$.
When these criteria are applied to the example spectra shown in Figure 4, ear-generated distortion products are detected in three cases as indicated by black arrows above the corresponding distortion frequencies. Two were found for listener L9 (top row, left column) at the harmonics $f_{3}$ and $f_{5}$; one was found for listener L8 (bottom row, left column) at the harmonic $f_{2}$. Listeners L4 and L6 (right column) had no ear-generated distortion products for the $4 \mathrm{~Hz}$ signal frequency. The SPLs of the three distortion products of L8 and L9 are well below those of the reference thresholds (black dashed lines in Fig. 4).

In all listeners, ear-generated distortion products were detected for at least one of the four infrasound frequencies. However, the occurrence of these distortions was not equally distributed across the four infrasound frequencies, as shown in Table 2. The table lists, for each of the four signal frequencies, $f_{1}$, the number of ear-generated distortion products found at the higher harmonics $f_{2}$ to $f_{9}$. Of all $16 \times 4 \times 8=512$ possibilities of observing an ear-generated distortion product, only 51 (i.e., less than $10 \%$ ) turned out to be true findings according to the criteria. Almost all had harmonic numbers between 2 and 5; only one was detected at the sixth harmonic of the $16 \mathrm{~Hz}$ signal and another one at the eighth harmonic of the $8 \mathrm{~Hz}$ signal. Most $(n=34)$ eargenerated distortion products were found for the $4 \mathrm{~Hz}$ and the $8 \mathrm{~Hz}$ signals. Seventeen of these were in the infrasound range (gray background in Tab. 2), and they belong exclusively to the $4 \mathrm{~Hz}$ signal. They are opposed to only three ear-generated distortions at the fifth harmonic of the $4 \mathrm{~Hz}$ signal, i.e., in the audio-frequency range. In general, distortion product levels were well below reference thresholds (at least $10 \mathrm{~dB}$ ) for all signal frequencies and all listeners. 
Table 2. Number of ear-generated distortion products found at the higher harmonics $f_{2}$ to $f_{9}$ of the signal frequency $f_{1}$, based on 16 individual measurements of the magnitude spectrum per frequency. Harmonics in the infrasound range are indicated in bold font.

\begin{tabular}{lccccccccc}
\hline Signal frequency & \multicolumn{10}{c}{ Number of distortion products at harmonic } \\
\cline { 2 - 10 } & $f_{2}$ & $f_{3}$ & $f_{4}$ & $f_{5}$ & $f_{6}$ & $f_{7}$ & $f_{8}$ & $f_{9}$ & $\sum$ \\
\hline $4 \mathrm{~Hz}$ & $\mathbf{8}$ & $\mathbf{3}$ & $\mathbf{6}$ & 3 & 0 & 0 & 0 & 0 & 20 \\
$8 \mathrm{~Hz}$ & $\mathbf{0}$ & 11 & 0 & 2 & 0 & 0 & 1 & 0 & 14 \\
$12 \mathrm{~Hz}$ & 0 & 7 & 0 & 0 & 0 & 0 & 0 & 0 & 7 \\
$16 \mathrm{~Hz}$ & 1 & 8 & 0 & 0 & 1 & 0 & 0 & 0 & 10 \\
$\sum$ & 9 & 29 & 6 & 5 & 1 & 0 & 1 & 0 & 51 \\
\hline
\end{tabular}
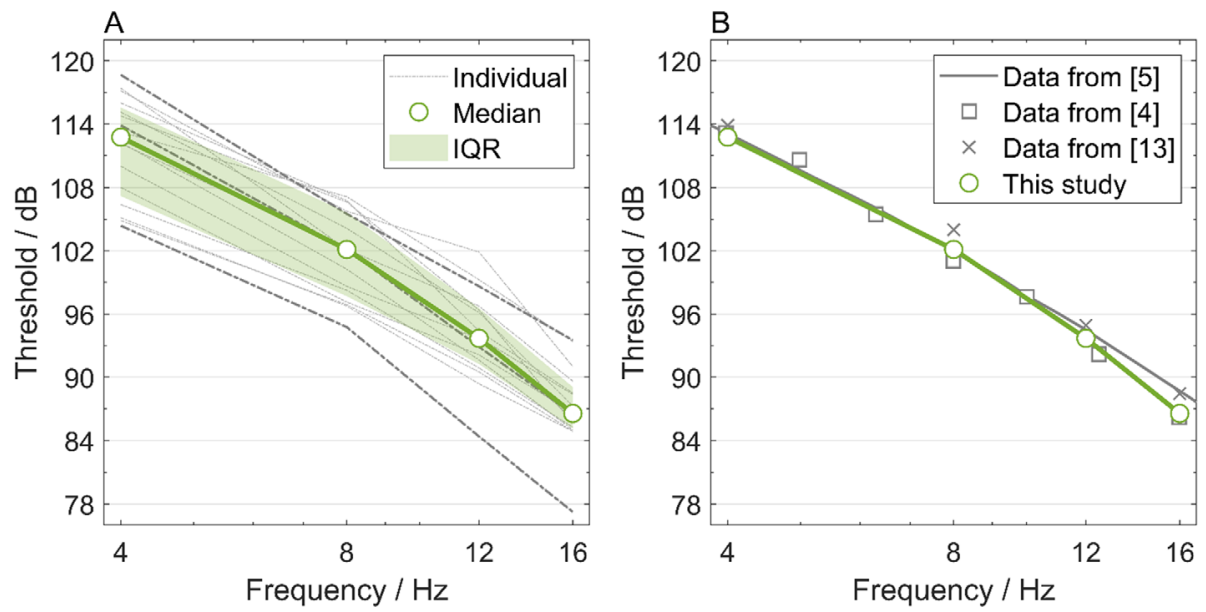

Figure 6. Threshold levels for infrasound stimuli. Panel A: Data for pure-tone infrasound stimuli. Green circles and the green semitransparent area show the median sound pressure level at threshold in $\mathrm{dB}$ and the corresponding interquartile range, respectively, across all 16 listeners. Gray dotted-dashed lines indicate individual thresholds; the slightly darker and thicker lines indicate thresholds of those three listeners, who had most ear-generated distortion products (see text). Panel B: The green circles are a reproduction of the median thresholds in Panel A. The gray solid line indicates the 2nd-order regression of reference thresholds reported in Figure 10 of [5]. The boxes represent threshold values reported in [4]. Crosses indicate pure-tone thresholds published in [13]; five out of their 14 listeners also participated in the present study.

\subsection{Correlation between thresholds and ear-generated distortion products}

Panel A of Figure 6 shows the mean SPL at threshold as a function of pure-tone frequency for each of the 16 listeners (gray dotted-dashed lines). Superimposed are the grand median (green circles) and the corresponding interquartile range (green semi-transparent area). Thresholds decrease monotonically from $112.8 \mathrm{~dB}$ at $4 \mathrm{~Hz}$ to $86.6 \mathrm{~dB}$ at $16 \mathrm{~Hz}$. Panel B of Figure 6 compares the grand median thresholds of this study with data from the literature (see Discussion section).

To test the hypothesis of a possible contribution of distortions to infrasound detection, the threshold data of the 16 listeners were separated for each frequency according to the absence and the presence of ear-generated distortion products at any higher harmonic from $f_{2}$ to $f_{9}$ of the signal frequency $f_{1}$.

Panel A of Figure 7 shows those individual thresholds, where no ear-generated distortion products were measured, with filled blue downward-pointing triangles. In addition, the median thresholds of this group are shown with open blue downward-pointing triangles connected with a solid blue line. The interquartile range is indicated by a semitransparent light blue area. Panel B of Figure 7 shows the individual thresholds where ear-generated distortion products were detected with filled orange upward-pointing triangles and the median thresholds of this group are shown with open orange downward-pointing triangles connected with a solid orange line; the interquartile range is indicated by a semi-transparent light orange area. For comparison, each panel also shows the median thresholds of the other group (with the corresponding symbol and connected with a dashed line).

The largest level difference between the two medians is observed at $4 \mathrm{~Hz}$. At this frequency, the median threshold for the listeners without distortions was $2.4 \mathrm{~dB}$ lower than the median threshold for those listeners where no distortions were detected. This difference is not significant (Wilcoxon rank-sum test with $p>0.8$ and $W=32$ ). The differences at the other three frequencies are considerably smaller $(0.1 \mathrm{~dB}$ at $8 \mathrm{~Hz}$ and $12 \mathrm{~Hz}$, and $0.8 \mathrm{~dB}$ at $16 \mathrm{~Hz}$; each Wilcoxon rank-sum test with $p>0.7$ and $W>35$ ).

Figure 8 compares the individual thresholds with the individual noise levels in quiet. The panels show the threshold as a function of background noise for each of the four 

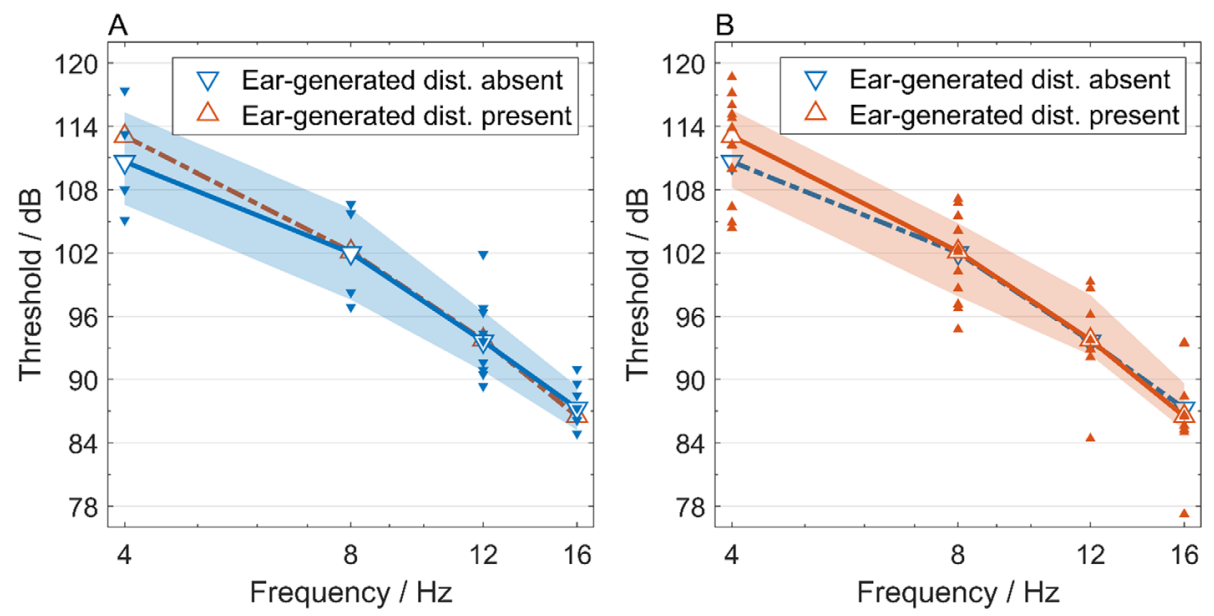

Figure 7. Sound pressure levels at threshold for pure-tone infrasound stimuli. The threshold data of the 16 listeners were separated according to the absence (blue, downward-pointing triangles) and presence (red, upward-pointing triangles) of ear-generated distortion products at any higher harmonic from $f_{2}$ to $f_{9}$ of the signal frequency $f_{1}$. Open triangles indicate the median thresholds for the two groups of listeners. Semi-transparent areas indicate the corresponding inter-quartile ranges for those with distortion products (Panel B) and those without (Panel A).

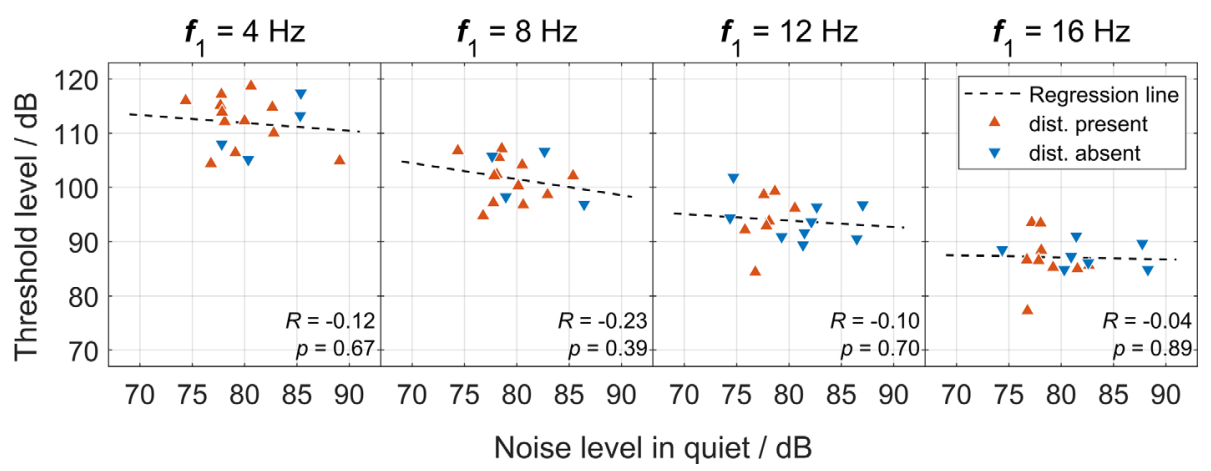

Figure 8. Analysis of correlations between infrasound thresholds and physiological infrasound noise in the ear canal. Each panel shows the analysis for one of the four infrasound frequencies. Each triangle represents the data of one of the 16 listeners. Colors and symbols indicate the absence (blue, downward-pointing triangles) or presence (red, upward-pointing triangles) of ear-generated distortion products at any higher harmonic from $f_{2}$ to $f_{9}$ of the signal frequency $f_{1}$. To quantify physiological infrasound noise, the average spectrum (average of five) was measured in quiet and integrated from $2 \mathrm{~Hz}$ to $20 \mathrm{~Hz}$. The integrated power is expressed in dB.

signal frequencies, $f_{1}$. The noise level was calculated for the infrasound-frequency range from $2 \mathrm{~Hz}$ to $20 \mathrm{~Hz}$ only, but the results do not change when the range is extended to low audio frequencies (not shown). There is a strong overlap between the data points for the group of thresholds with ear-generated distortion products (red, upward-pointing triangles) and those without ear-generated distortion products (blue, downward-pointing triangles).

\section{Discussion}

The aim of the present study was to test the hypothesis that audible ear-generated distortion products are involved in infrasound perception. A custom-made low-distortion sound reproduction system, LDREPS [11], was used to detect ear-generated distortion products in the ear canal and to measure infrasound thresholds in quiet. In all listeners, ear-generated distortion products were detected for at least one of the four infrasound frequencies, indicating a non-linear response to these infrasound stimuli, but their levels were well below reference thresholds. Note that, at this point, it is unclear, which mechanism causes the eargenerated distortion products for the infrasound stimuli. This contrasts to otoacoustic emissions (OAEs, [9]), where it is commonly assumed that their origin is an active process within the cochlea (e.g., [17]). A key part for OAEs seems to be that sound is propagating backwards from the cochlea to the ear canal, where it is then finally recorded. For infrasound, the origin could still be in the cochlea; but it is also possible that, due to the high SPLs, non-linear properties of other parts of the ear, such as the middle ear, or the eardrum, play a role. The level measured in the ear canal may differ from that acting in the inner ear. Thus, levels of distortion products below threshold may not necessarily be seen as evidence against the hypothesis of infrasound 
detection through audible distortion products, at least if they are not generated in the outer ear. If the source of the measured distortions was solely the outer ear, they would be inaudible, since their levels were well below threshold.

To test a possible contribution of distortion products to infrasound detection, the thresholds were related to the analysis of the sound field in the ear canal. The grand median thresholds of this study (measured with LDREPS) are similar to thresholds reported in the literature (measured with other setups). The maximum deviation between the different data sets shown in Panel B of Figure 6 is $3 \mathrm{~dB}$, i.e., well within the interquartile range of the present data (Panel A of Fig. 6).

In general, the data did not support the hypothesis of ear-generated distortions contributing to infrasound perception. The listeners with ear-generated distortion products for a specific frequency did not have lower thresholds than those without (see Fig. 7). The $4 \mathrm{~Hz}$ thresholds for listeners with distortion products for this signal frequency were even slightly higher than those for listeners without distortions, although the difference was not significant. For all other frequencies, thresholds for listeners with distortion products were about the same as those for listeners without distortion products. Thus, the presence of ear-generated distortion products seems to have no clear impact on pure-tone thresholds of infrasound stimuli in quiet. This could have already been expected on the basis of the individual data shown in Figure 4. The two listeners in the top panels had very high thresholds and the two listeners in the bottom panel had very low thresholds. In each group of listeners, one did have ear-generated distortions and one did not.

The two example listeners of Figure 4 not showing eargenerated distortion products had higher physiological noise than the two showing ear-generated distortion products. Thus, one may hypothesize that ear-generated distortion products were only detected when the physiological noise was low. To test this hypothesis and answer the question if infrasound thresholds are determined by the physiological background noise, the individual thresholds were compared to the individual noise levels in quiet (see Fig. 8). This analysis indicated that thresholds do not correlate with physiological background noise: For all signal frequencies, the absolute value of the Pearson correlation coefficient, $R$, is low $(|R|<0.3)$. Thus, ear-generated distortion products are detected not only in listeners with low levels of physiological noise.

Considering the absence of correlation between distortion products, thresholds, and physiological noise, the present data suggest that distortion products may not play a key role in infrasound perception. This is also supported by a recent publication of Marquardt and Jurado [18]. They found that it is difficult to distinguish an amplitude-modulated tone from a tone with an additional infrasound, indicating that infrasound is perceived as modulation. Since there is always a certain residual background noise in the ear (due to, e.g., blood flow), one may, e.g., argue that infrasound is always perceived as modulation (of this residual noise when the infrasound is presented in quiet).

\section{Conclusions}

The present study used a low-distortion sound reproduction system to detect ear-generated distortion products in the ear canal for infrasound pure-tone stimuli at suprathreshold SPLs. Ear-generated distortion products were detected in all of the listeners for at least one of the signal frequencies, indicating a non-linear response to these infrasound stimuli. However, the SPLs of the ear-generated distortion products were considerably lower than the reference threshold levels for their frequencies (more than $10 \mathrm{~dB}$ ). In addition, thresholds of listeners with ear-generated distortion products for a specific frequency did not have lower thresholds than those without distortions for this frequency. These findings suggest that higher harmonics do not provide detection cues for infrasound pure tones.

\section{Acknowledgments}

The project was supported by the Deutsche Forschungsgemeinschaft (DFG, projects FE 1192/3-1 and VE 373/4-1). Special thanks go to all the listeners who participated in the experiment.

\section{References}

1. ISO 7196:1995: Acoustics - Frequency-weighting characteristic for infrasound measurements. International Organization for Standardization, Geneva. https://www.iso.org/obp/ ui/es/\#iso:std:iso:7196:ed-1:v1:en.

2. N.S. Yeowart, M.J. Evans: Thresholds of audibility for very low-frequency pure tones. Journal of the Acoustical Society of America 55, 4 (1974) 814-818.

3. G. Békésy: Über die Hörschwelle und Fühlgrenze langsamer sinusförmiger Luftdruck schwankungen. (On the hearing threshold and the sensation limit of sinusoidal air pressure fluctuations). Annalen der Physik 26, 5 (1936) 554-566.

4. R. Kühler, T. Fedtke, J. Hensel: Infrasonic and low-frequency insert earphone hearing threshold. Journal of the Acoustical Society of America 137 (2015) EL347-EL353.

5.H. Møller, C.S. Pedersen: Hearing at low and infrasonic frequencies. Noise Health 6 (2004) 37-57.

6. O. Behler, S. Uppenkamp: Activation in human auditory cortex in relation to the loudness and unpleasantness of lowfrequency and infrasound stimuli. Plos One 15 (2020) e0229088. https://doi.org/10.1371/journal.pone.0229088.

7. C. Jurado, T. Marquardt: Brain's frequency following responses to low-frequency and infrasound. Archives Acoustics 45 (2020) 313-319.

8. M. Weichenberger, R. Kühler, M. Bauer, J. Hensel, R. Brühl, A. Ihlenfeld, B. Ittermann, J. Gallinat, C. Koch, T. Sander, S. Kühn: Brief bursts of infrasound may improve cognitive function - An fMRI study. Hearing Research 328 (2015) 8793.

9. D.T. Kemp: Stimulated acoustic emissions from within the human auditory system. Journal of the Acoustical Society of America 64 (1978) 1386-1391.

10. E. Burke, J. Hensel, T. Fedtke, S. Uppenkamp, C. Koch: Detection thresholds for combined infrasound and audiofrequency stimuli. Acta Acustica united with Acustica 105 (2019) 1173-1182. 
11. H. Joost, B. Friedrich, J.L. Verhey, T. Fedtke: How to present pure-tone infrasound to the ear. Proceedings of the 23rd International Congress on Acoustics, Aachen, 2019, pp. 1470-1477. http://pub.dega-akustik.de/ICA2019/data/articles/000733.pdf.

12. ISO 389-7:1996: Acoustics - Reference zero for the calibration of audiometric equipment - Part 7: Reference threshold of hearing under free-field and diffuse-field listening conditions. International Organization for Standardization, Geneva.

13. B. Friedrich, H. Joost, T. Fedtke, J.L. Verhey: Spectral integration of infrasound at threshold. Journal of the Acoustical Society of America 147 (2020) EL259-EL263.

14. H. Levitt: Transformed up-down methods in psychoacoustics. Journal of the Acoustical Society of America 49 (1971) $467-477$.
15. ISO 389-9:2009: Acoustics - Reference zero for the calibration of audiometric equipment - Part 9: Preferred test conditions for the determination of reference hearing threshold levels. International Organization for Standardization, Geneva.

16. R.A. Dobie, M.J. Wilson: A comparison of t test, F test, and coherence methods of detecting steady-state auditory-evoked potentials, distortion-product otoacoustic emissions, or other sinusoids. Journal of the Acoustical Society of America 100 (1996) 2236-2246.

17.D.T. Kemp: Otoacoustic emissions, travelling waves and cochlear mechanisms. Hearing Research 22 (1986) 95-104.

18. T. Marquardt, C. Jurado: Amplitude modulation may be confused with infrasound. Acta Acustica united with Acustica 104 (2018) 825-829.

Cite this article as: Joost H, Friedrich B, Verhey JL \& Fedtke T. 2021. Is infrasound perceived by the auditory system through distortions? Acta Acustica, 5, 4. 